\title{
FIRST PANEL DISCUSSION
}

[On the last day of this meeting two general discussions were held, each beginning with comments by members of a discussion panel. The first panel and discussion concentrated on reviewing the theoretical problem of the LBV eruption mechanism. Members of this panel were I. Appenzeller, C. de Jager, D.G. Hummer, F.D. Kahn, and H.J.G.L. M. Lamers, with K. Davidson acting as moderator or chairman. Remarks by panel members and other participants are given here in condensed form, somewhat reworded for brevity and clarity. It will be noted that the most generally accepted consensus here was that the basic cause of LBV instability is not yet known!]

Appenzeller: Having the privilege of opening this discussion, I shall start with a brief overview and a personal assessment of the different physical processes that have been discussed during this meeting as possible causes of the peculiar properties and dramatic variabilities of the LBV's. To follow some logical order, stellar interior mechanisms will be dealt with first, followed by atmospheric and circumstellar phenomena.

Among the interior mechanisms, we first have the suggestion of multi-mode pulsations induced and powered by interior differential rotation. Sreenivasan has outlined the details of this scenario in his contribution to this volume. They are complex and depend upon the interior structure. Therefore it is difficult to derive a reliable observational signature that could be used to prove the presence of this mechanism.

Another potential deep-interior process involves thermal oscillations as described by Norbert Langer for hydrogen shell burning models. Of course this suggestion depends critically on the preceding evolutionary phases and a star's ability to reach the stage in question.

As Maeder pointed out, a density inversion observed in computed stellar models near the red boundary of the LBV region in the H-R diagram may indicate the onset of violent events at an earlier evolutionary stage. Because of Rayleigh-Taylor instabilities it is very unlikely that density inversions will occur in real stars; but it is not clear how a real star will react to a density gradient trying to change its sign. Strongly enhanced mass loss as observed in LBV eruptions could well result, but a milder reaction (e.g., modified convection properties) also seems possible. Obviously this phenomenon deserves further studies.

From the discussions at this meeting it seems that radiation-pressure-induced massloss instabilities are still the most popular concept for explaining LBV eruptions. The new, sophisticated model computations reported by Kudritzki et al., Leitherer et al., and by Owocki seem to provide further support for this type of mechanism. However, only time-dependent computations could actually prove that this mechanism is indeed the cause of the observed variations.

As described in de Jager's review talk, turbulent pressure effects must be present in practically all extremely luminous stars. However, at least in the hotter LBV's (and during minimum phases) radiation pressure probably dominates and turbulent or wave pressure probably can become important only in conjunction with radiation effects. 
Another mechanism that can explain certain observed properties of the LBV's is rapid mass exchange in massive close binary systems (see Gallagher's excellent review). But, in spite of many high-quality observational results which have been accumulated for the known S Dor stars, in no case has reliable evidence for a close binary nature been detected. If a typical LBV is a binary system, the components must be hidden in common envelopes. But the minimum-state spectra of objects such as $R 71$ are hardly compatible with this assumption.

Summarizing, I personally feel that radiation pressure instabilities are the most likely cause of the LBV variability. However, additional work will be needed to identify the physical mechanism leading to the LBV eruptions without ambiguity.

De Jager: First a few words on very large explosions à la Eta Carinae. That 'explosion' lasted for about ten years and caused a mass ejection of the order of two solar masses. Since such an ejection involves about half the stellar volume, the disturbance leading to it should have been seated somewhere well outside the stellar center but well below the surface. It is remarkable that we have a theory of ordinary novae (atmospheric explosions involving about 0.0001 solar mass) and of supernovae (core explosions involving nearly the whole stellar mass) but not of these slow intermediate-scale events.

With regard to the regular LBV ejection events my ideas are the following. We know that the stars in the upper part of the H-R diagram are pulsating with periods of $\sim 4$ to $\sim 50$ days in the LBV region (depending on spectral type and luminosity). The pulsations may be an ordered system of non-radial pulsations or they may have a more stochastic character. The pulsations are gravity waves. An important point is that LBV's differ from other stars in the same part of the H-R diagram in that their pulsations do not show high-frequency components, and have larger amplitudes (judging from the photometric variations). We combine this observation with the fact that the upper limit of the LBV area in the H-R diagram coincides with the line where the stellar wind velocity equals that of sound. Only a relatively small additional (pulsational) outward acceleration of part of the stellar surface would then be sufficient to shift the locus of $(v /$ sound speed $)=1$ inward to fairly dense photospheric layers, thus dramatically increasing the local rate of mass loss. In my talk at this meeting I gave examples. Therefore I think that the stellar non-radial (or stochastic) pulsations are responsible for the mass ejections. If the pulsations are really stochastic, then larger amplitudes may occasionally occur, causing larger ejections.

Hummer: At the beginning of this meeting we heard a quotation from John Herschel relative to the LBV's which included the phrases "fitfully variable to an astonishing extent" and "having no regularity of progression." I would like to speculate on a mechanism that may have a bearing on these phenomena.

Both the Munich and the JILA groups have reported here the operation of what might be called the 'ionization instability' in LBV's. A slight decrease in the degree of ionization of hydrogen in response to some small change in one or more parameters leads to an increase in the opacity in the Lyman continuum, which then causes further recombination and yet further increases the optical thickness, until the hydrogen is essentially neutral ( $c f$. the Strömgren sphere). As the Lyman continuum becomes opaque, the dominant lines driving the stellar wind switch from Fe III to Fe II and the 
mass loss rate increases. In the particular case of $\mathrm{P} \mathrm{Cyg}$, the Munich group showed that varying the inner radius of the atmosphere by as little as $1.5 \%$ triggered this mechanism.

This instability is an example of a nonlinear amplifier, in which the output remains small until the input reaches some threshold value, beyond which the output suddenly increases. If the output above threshold is roughly constant, we speak of a "bistable" device.

What is the 'input' in the case of P Cyg? I suggest that it could be fluctuations of the radius of the base of the photosphere caused by non-radial pulsations. Although theory at present neither predicts nor forbids non-radial pulsations for LBV's, we do know that they flicker on the level of a tenth of a magnitude. Small irregular variations in radius at any point of the stellar surface would have little effect so long as their amplitude is smaller than some threshold value, which is determined by the stellar parameters. If the star is unstable to non-radial pulsations in many modes, then at a given point on the surface the various modes would interfere destructively and nothing would happen. But if occasionally they interfered constructively, the threshold amplitude could be surpassed and a major change in the local atmospheric structure would occur.

Two consequences are implied. First, the characteristic times between outbursts would be much longer than those of the pulsations. Secondly, the size of the ouburst would scale with the area of coherent fluctuations. Thus very large outbursts, covering much or all of the surface, could be rare compared to smaller ones. Localized outbursts would give rise to the formation and ejection of clumps of matter.

Kahn: It has been remarked that a large outburst by a star like P Cyg releases an amount of radiant energy of order $10^{49} \mathrm{ergs}$ (and a similar amount in mechanical energy), and might therefore be regarded as being in some way analogous to a supernova explosion in which the prompt release of radiant energy is rather similar.

Here I want to consider this view a little more deeply, because there are also very obvious differences between supernova explosions, on the one hand, and big LBV eruptions on the other. The total energy release in a Type II SN is of order $10^{53}$ ergs, including neutrinos, and of order $10^{51}$ ergs including only baryonic material. Simply making a comparison between the energy released in the optical and UV near the time of the event therefore gives a misleading impression: the major release of energy in a supernova, apart from the neutrinos, is in the form of a violent explosion of material with typical speeds of thousands of $\mathrm{km} / \mathrm{s}$. The eruptions of LBV's do not have a comparable counterpart.

There is another big difference between the two phenomena. In the case of a supernova the release of energy takes place essentially in a fraction of a millisec deep inside the star and the effects reach the surface within a few hours. In the case of an LBV the violent eruptions produces a continuing disturbance which lasts for years, and it is therefore much harder to regard it as a single event, since the subsequent response goes on for such a long time.

Nevertheless $10^{49}$ ergs is a great deal of energy, even for a massive star, almost of the order of its binding energy. In its present state the star radiates this amount in about a century or so. Whatever was the unknown process that led to this event, it must have taken place deep down inside the star. The present manifestations of LBVdom are of course much milder, and consist of a massive wind driven of $f$ at modest 
speed together with irregular fluctuations in photospheric radius. To me the simplest view is that we are still, nevertheless, witnessing the consequences of some physical state that gave rise to the violent eruption. The star has reached a state in its evolution where no stable static structure is available to it. Consequently, an unstable disturbance has grown to finite amplitude somewhere deep in its interior. The resulting oscillations are rumbling away in an irregular and probably unpredictable fashion and are transmitting their energy in the form of pressure waves to the surface. The deposition of mechanical energy there is, in my view, the primary cause of the expansion of the wind. It is only to be expected that such a wind will be irregular on a variety of timescales. The fluctuations in photospheric radius and in visual magnitude are an inevitable consequence. The formation of shells in the wind at some distance above the star is another.

Lamers: The high luminosities and low effective gravities of the LBV's suggest that radiation pressure may play a key role in their behavior. Several speakers at this colloquium have discussed the possible effects of radiation pressure for explaining the high and variable mass-loss rates of LBV's. I would like to make a few remarks about the connection between radiation pressure and the evolution of massive stars.

Stellar surface layers where $\kappa_{\mathrm{F}} \geq 4 \pi G M c / L$ (where $\kappa_{\mathrm{F}}$ is flux-mean opacity) will not be bound by gravity. The opacity depends on density, temperature, and composition. For Population I stars, $\kappa_{F}$ in the photosphere has a maximum near $T_{\text {eff }} \approx 10000 \mathrm{~K}$ and decreases for higher and lower $T_{\text {eff. }}$. This implies that the luminosity in the H-R diagram where the photospheres become unbound will reach a minimum $L$ at $T_{\text {eff }} \approx$ $10000 \mathrm{~K}$ and will increase to higher or lower $T_{\text {eff. }}$. Lamers and Fitzpatrick (1988, Astrophys.J. 324, 279) suggested that this can explain the Humphreys-Davidson limit.

[The idea was also described, e.g., in Science 223, 227 (1984).] In reality the outer layers may become unstable before $L$ reaches the limit set by $\kappa_{\mathrm{F}}$, because a looselybound atmosphere with a very small effective gravity may easily become unstable by secondary effects such as pulsation or turbulence. It may very well be that such secondary processes trigger the shell ejections in LBV's, but the major reason for their instability is still radiation pressure. I doubt that such secondary effects can trigger the large eruptions such as the $\eta$ Car event, because the large amount of energy involved in such an event requires a more efficient mechanism operating deep below the photosphere.

If the LBV variability is due to triggering at small $g_{\text {eff }}$, then there are two ways to explain the co-existence of LBV's and normal supergiants in the same part of the H-R diagram. Either the more normal stars have higher masses and larger gravities than LBV's with similar luminosities, or the triggering is less efficient, or both. Lower masses for LBV's require that they have lost more mass than the normal stars. This agrees with their higher nitrogen abundances. Maybe the LBV's were more rapid rotators and lost more mass on the main sequence, but this is very difficult to test observationally. Or maybe they have lost more mass because they have suffered one or more large eruptions already. In that case, shouldn't we expect ring nebulae around most LBV's?

The question of triggering may have consequences for the formation of $B[e]$ stars. These differ from LBV's in that they have most of their mass loss in equatorial (?) disks. This idea is similar to the classical Be stars, which are on the average fast rotators, but not fast enough to produce sufficient centrifugal forces. The equatorial ejection is supposed to be due to the combined effects of non-radial pulsation and 
radiation pressure. Rotation is important for creating non-radial pulsations with their highest amplitudes along the equator. Maybe a similar mechanism is operating in the $\mathrm{B}[\mathrm{e}]$ stars with low $\boldsymbol{g}_{\text {eff }}$ and some triggering along the equator? As in the case of the Be stars, one does not need a large rotational velocity to eject in a disk-like fashion.

A critical test for the role of radiation pressure in the explanation of the LBV and $\mathrm{B}[\mathrm{e}]$ phenomenon can be made by comparing their locations in the $\mathrm{H}-\mathrm{R}$ diagram for galaxies with different metal abundances. Since $\kappa_{F}$ will decrease with decreasing metallicity, one might expect that the LBV's in low-metallicity galaxies occur at higher luminosities than the galactic ones. One should keep in mind, however, that the luminosity of the LBV's will not only depend on $\kappa_{F}$ but also on the $M / L$ ratio, which may be different in low-metallicity galaxies.

\section{General discussion:}

Shore: In theoretical astrophysics various dimensionless numbers are useful or interesting. Here we have one-- we might call it the Eddington number: $\Gamma<1$ could play a role like (for instance) the Rayleigh number $(>1)$ that pertains to convection, with a phase transition. In the recombination mechanism we have a non-linear instability which may have 3 time-dependent equations connecting ionization, mass in the envelope, and velocity, all as functions of time. In dynamo theory, e.g., that type of structure leads to a phase transition with lots of noise before it flips; it is the prescription for disaster because it's the signal of a Strange Attractor. We may be dealing with something that has a bistable character, flips back and forth, and does so because the instability that is driving it is intrinsically non-linear, buried fairly deep in the envelope but not in the core ... but if there is a strange attractor, if this is a bistable system, then we may never find what triggers it all; it's simply intrinsically unstable and the spectrum of flips may be unpredictable. I might also add that this could drive a close companion star over the edge of sanity, too.

Sreenivasan: ... Regarding the trigger for LBV explosions -- The facts that many modes are excited in non-radial pulsations and that their frequencies are close suggest modecoupling. This gives rise to flickering at higher frequencies and a modulation at lower frequency. Occasionally, the amplitudes could add constructively and push the overlying material off at the escape velocity which has been lowered by radiation pressure, mass, and centrifugal force. Just when the trigger gets pulled cannot be predicted and this could well look like 'deterministic chaos' as Steve Shore remarked.

Davidson: Some of us have been wondering why non-linear behavior wasn't emphasized more at this meeting! As Hummer and Shore implied, even Herschel's and Clerke's choices of words 90 to 150 years ago ring chaotic, non-linear alarm bells when we read them today.

... About another matter: Henny Lamers referred to the effect of metallicity on an opacity-dependent instability. Here we have to be careful about what's conspicuous in an empirical H-R diagram, and what would not be. Suppose that the Upper Limit is lower for stars with higher heavy-element abundances. Then the Upper Limit that we notice in the diagram would really be an envelope representing the lowest metallicities that happen to be observationally accessible. Some stars of higher metallicity might be marginally unstable or might be LBV's well below that envelope. (Maybe this is pertinent to Katy Garmany's talk. Also, this should remind us that an Upper Limit, a locus of instability, and a locus of LBV behavior can logically be three different lines that need not coincide.) The stability boundary for stars with nearly primordial composition may be higher, but I Zw 18 isn't close enough to check! 
Humphreys: We have reasonable data for just three galaxies: the Milky Way, LMC, and SMC. They do have different metallicities, but in the SMC, with the lowest metallicity, the sample size is so small that there are almost no very massive stars. Population statistics may dominate any effect due to metallicity differences. It would be nice if we could examine a big spiral galaxy with low metallicity.

Gallagher: With the possible exception of $\eta$ Car, for each LBV we see evidence of only one really major ejection event. It would be good to look for additional faint outer shells; meanwhile, conceivably a major outburst is a one-time event. Are we perhaps being premature in assuming that LBV's live long enough to represent a signif icant mass-loss phase in the evolution of very massive stars?

Lamers: Indeed we don't know. Unfortunately, we know too few LBV's for a thorough statistical study. Major outbursts may occur on long timescales, possibly longer than $10^{4}$ years.

Walborn: I am not sure that a distinction between normal and "Plinian" outbursts is well established observationally. Does the latter refer only to $\eta \mathrm{Car}$, or does it include the 3-mag ranges of P Cyg and R 127? The difference may be only that $\eta$ Car is more massive and hotter than the other objects, and thus has a larger bolometric correction.

De Groot: We speak of P Cyg's outburst as a 3-magnitude event, but we do not really know this. There are no observations from before the year 1600 . The 3-mag amplitude is based on the star's behavior after that time, when an outburst was seen at maximum. The subsequent brightness of $\mathrm{P} \mathrm{Cyg} \mathrm{may} \mathrm{be} \mathrm{at} \mathrm{some} \mathrm{intermediate} \mathrm{level}$ and might not represent the pre-outburst brightness.

Davidson: We've been told of many indications of bipolar structure around LBV's. Maybe the existence of an axis of symmetry implies that some sort of angular momentum is important. Any comments on bipolarity?

Gallagher: Of course bipolarity might be an indication of binarity. This kind of structure may be important this way because it is so easy for other evidence of close binaries to be hidden.

Appenzeller: A bipolar outflow geometry cannot prove the binary nature of its source, because rotation or a magnetic field can define a preferred axis in single stars also. In fact, the strikingly well-collimated outflows from $T$ Tauri stars are now generally supposed to be single-star phenomena. It would be extremely interesting if one could learn more about the relatively sketchy indications of binarity, with observations like those described at this meeting by Paresce. Then one might start making use of this potential source of information.

Friedjung: I would like to make a comparison with classical novae. There is evidence for continued ejection driven by an object above the Eddington limit. In the nova case there may be an extra source of energy in outer layers which brings them above above the Eddington limit, but does not make the whole star fly away into space. Is something similar possible in LBV's, at least for the major outbursts?

Kahn: What you need is a layer of limited extent, deep down, where $\Gamma$ exceeds unity. This layer is unstable but cannot lift off all the overlying material. It must therefore oscillate at finite amplitude, and so generate waves which ultimately reach the surface of the star and drive a wind from there.

Maeder: It has been said that the layers above the $\Gamma=L / L_{\mathrm{Edd}}$ level may be ejected. Indeed, the amount of ejecta can be larger, since the critical level may move inward 
during the episode of shell ejection. In view of wide differences between stars such as $\eta$ Car and Var A, we may suspect that there is perhaps more than one unique mechanism helping to accelerate the exterior layers outward in very massive stars.

Hummer: During the past few days, many people have been discussing $\Gamma=g_{\mathrm{rad}} / g_{\mathrm{grav}}$ as though it were a constant parameter of each star. But $\Gamma$ depends on radius and must be less than unity deep in the star. It increases with radius and if it reaches unity, that layer is unstable and starts to move outwards. Just where this happens depends on how the opacity and radiative flux vary with location and frequency. In every hot star, $\Gamma$ reaches unity at the base of the stellar wind.

De Jager: For stars at the H-D limit, $\Gamma \approx 1$ at $\tau \approx 1$ for temperatures above $10^{4} \mathrm{~K}$. In some regions $\Gamma$ can decrease outward. This situation (unstable inside, more stable outside) has interesting hydrodynamic consequences.

Davidson: If we don't care about the normal wind and look only at optical depths of the order of unity or somewhat larger (averaged appropriately over frequency, of course), then $\Gamma$ in that region can characterize a star if we are careful with definitions. That was one point of an empirical remark I made earlier: In this sense $\Gamma=1$ does not resemble the observed Upper Limit in the H-R diagram, but rather is too level; for a hint about why, see John Castor's comment following my contribution in the book from the Lunteren meeting. (When examined closely, Lamers and Fitzpatrick's results do not contradict this assertion.) But $\Gamma=$ (some number around 0.8 ) in the same region does give a fit to the observed Limit. Why? -- Who knows!

Schmutz: Most people here seem to be happy with the instability found by the Munich group. I am not so happy, because I do not think it is a mechanism that can explain $\mathrm{S}$ Dor-type variations. There is no doubt that a change of iron ionization Fe III/Fe II is needed to explain the large difference between mass-loss rates at maximum and minimum (see also Leitherer $e t$ al., these proceedings). But the result found by the Munich group is an instability only if the stellar parameters are close to the critical ones. The observed temperatures and mass-loss rates of R 71, AG Car, and R 127 in their minimum states are far from being critical for Fe III to recombine! The stars must change their radii by large amounts before the critical values are reached.

Appenzeller: Interpreting the new improved model atmosphere computations, one has to keep in mind that so far these results have been obtained for only a rather limited set of parameters. The very important 'unified' P Cyg models all assume the same fixed effective temperature $(19000 \mathrm{~K})$ and thus provide no direct information on the temperature dependences of such atmospheres. Such calculations for an extended set of parameters would obviously be useful.

Kudritzki: For most of this meeting we have been depending on just apparent brightnesses and temperatures estimated from energy distributions. We have almost no good hard numbers based on quantitative spectroscopy of LBV's; we know a little about the abundances in ejecta, but do not have much data on atmospheric or photospheric abundances or on gravities (except for one object). So I think that it is necessary to work out observational techniques to give good numbers. For example, high members of the Balmer series in absorption can be used to determine gravities; high$\mathrm{S} / \mathrm{N}$ absorption line measurements can give $\mathrm{CNO}$ abundances; etc. So far we have little to work on!

De Jager: I agree. It would also be very helpful to monitor radial velocities as well as the brightness of at least one selected LBV for at least half a year. That would make it possible to study its internal structure and to decide on its evolutionary status. For comparison one should also monitor a non-LBV star at the same location in the H-R diagram. Excellent data on one LBV is better than partial data on many. 
Davidson: The need for more data is indisputable. Remember, though, that the paramount question about LBV's today is why they are so unsteady -- what triggers their eruptions and what supplies the energy for such events? It seems to me that our ignorance along these lines is due primarily to a lack of theoretical studies and only secondarily to a shortage of data (we have many observational clues even if they are not precise). At the same time, the unsteadiness of LBV's is in itself a significant constraint on their structure, if only we can understand the theory! What I'm trying to say is that this is a question that theorists can legitimately attack now, without having to wait for the improved observations.

Hillier: A comment to emphasize Roberta's point about low statistics. Here we have 5 panel members, and a 6 th if we include Kris Davidson, the session chairman. After two had spoken I noticed a correlation, that their surnames begin with letters in the first half of the alphabet. More observations confirm that all 6 LBV's share this correlation. Is this significant? -- Selection effects may occur and we must examine the population from which they were drawn. A quick count by Allan Willis suggests that the theorists were drawn from a biased sample but observationalists were more uniformly distributed.

'Panel 1'

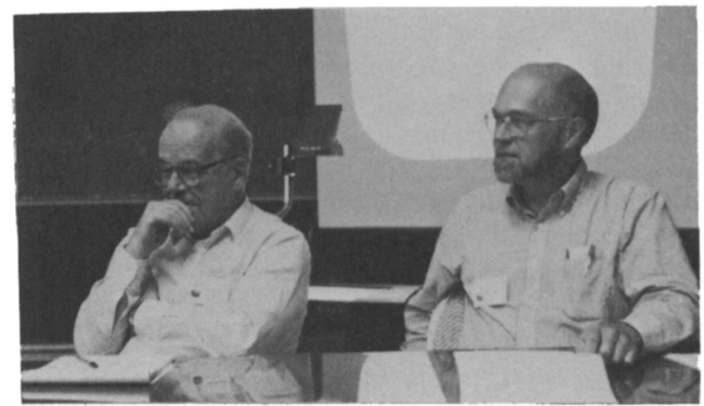

Kahn, Hummer

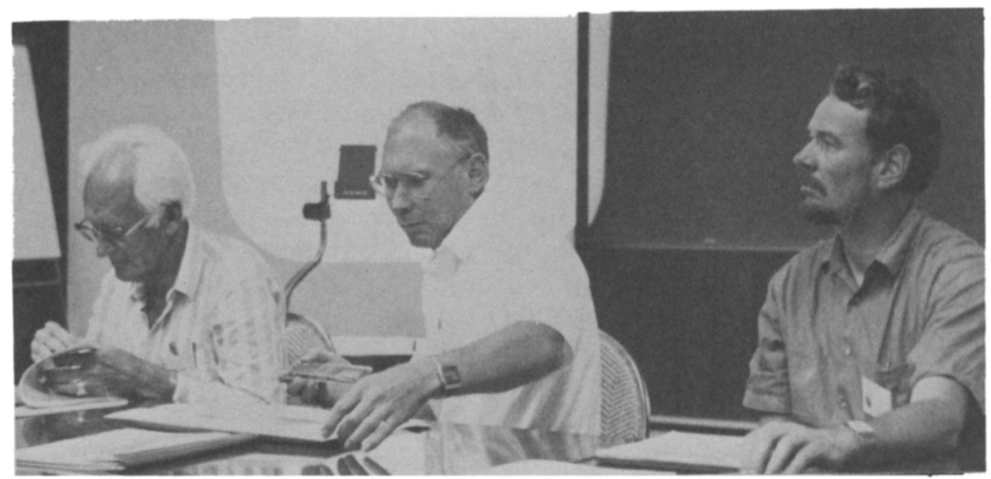

de Jager, Appenzeller, Lamers 